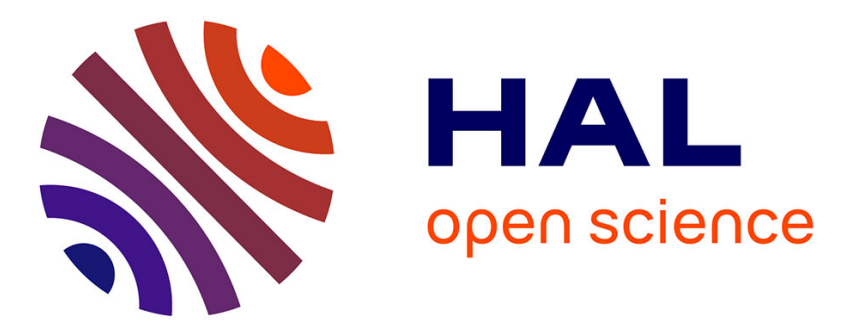

\title{
Torque in Permanent Magnet Couplings: Comparison of Uniform and Radial Magnetization
}

Romain Ravaud, Guy Lemarquand, Valérie Lemarquand, Claude L. Depollier

\section{To cite this version:}

Romain Ravaud, Guy Lemarquand, Valérie Lemarquand, Claude L. Depollier. Torque in Permanent Magnet Couplings: Comparison of Uniform and Radial Magnetization. Journal of Applied Physics, 2009, 105, pp.053904. 10.1063/1.3074108 . hal-00366546

\section{HAL Id: hal-00366546 https://hal.science/hal-00366546}

Submitted on 9 Mar 2009

HAL is a multi-disciplinary open access archive for the deposit and dissemination of scientific research documents, whether they are published or not. The documents may come from teaching and research institutions in France or abroad, or from public or private research centers.
L'archive ouverte pluridisciplinaire HAL, est destinée au dépôt et à la diffusion de documents scientifiques de niveau recherche, publiés ou non, émanant des établissements d'enseignement et de recherche français ou étrangers, des laboratoires publics ou privés. 


\title{
Torque in PM Couplings: Comparison of Uniform and Radial Magnetization
}

\author{
R. Ravaud, G. Lemarquand, V. Lemarquand and C. Depollier
}

\begin{abstract}
We present a three-dimensional study of the torque transmitted between tile permanent magnets uniformly magnetized. All this study is based on the Coulombian model. The torque is calculated semianalytically by considering all the surface densities that appear on the tiles. In addition, no simplifying assumptions are done in the expressions given in this paper. Consequently, the evaluation of the torque is very accurate and allows us to show the drawbacks of using tile permanent magnets uniformly magnetized instead of using tile permanent magnets radially magnetized. Such an approach is useful because it allows us to realize easily parametric studies.
\end{abstract}

\section{Index Terms}

Magnetic coupling, Tile permanent magnet, Torque, Uniform magnetization, Radial magnetization, Three-dimensional calculation.

${ }_{13}$ AGNETIC couplings are often realized with tile permanent magnets radially or uniformly ${ }_{14} 1$ magnetized. Tile permanent magnets radially magnetized allow us to obtain great couplings 15 but they are difficult to fabricate. Consequently, an alternative experimental method consists in using 16 tiles uniformly magnetized. Indeed, they are simpler to fabricate than tiles radially magnetized and thus 17 cheaper. Unfortunately, they are also less efficient and can lower the quality of transmission between tiles 18 located on the stator and tiles located on the rotor. Therefore, it is interesting to predict theoretically the 

40 magnetized.

way the torque is transmitted between tile permanent magnets uniformly magnetized. Such a model allows

us to realize easily parametric studies and thus to know when the use of tile permanent magnets radially magnetized is necessary or not. Indeed, the angular width has a great influence on the radial field created by a tile permanent magnet uniformly magnetized. As this radial field is not perfectly symmetrical, the torque transmitted is modified. Therefore, the aim of this paper is to determine precisely with a three-dimensional model how the torque transmitted between tiles changes according to the way the tiles are magnetized. Many authors have studied magnetic couplings [1]-[4] and structures using permanent magnets [5]-[10]. Historically, we can say that the first models used to study magnetic couplings were the two-dimensional models [11]-[19]. The main reason lies in the fact that a two-dimensional approach is fully analytical and allows us to make an easy parametric optimization of the permanent magnet dimensions. However, this 2D-approach is not valid when we determine the magnetic field far from one magnet [20].

Tree-dimensional approaches seem to be more difficult to realize parametric studies because they are not fully analytical. In fact, the calculation of the radial and axial magnetic field components created by tile permanent magnets is not strictly analytical but is necessary based on special functions [21]- [40].

More generally, all the semi-analytical or analytical approaches used by several authors allow manufacturers to optimize devices using permanent magnets [41]-[48].

This paper presents a semi-analytical approach based on the Coulombian model for calculating precisely the torque transmitted between tile permanent magnets uniformly magnetized. This semi-analytical approach is three-dimensional. We explain in the second section how this problem can be solved. Then, we present a semi-analytical expression of the torque transmitted between two tile permanent magnets uniformly magnetized. Eventually, we present the main drawbacks of tile permanent magnets uniformly

\section{Modeling Tile Permanent Magnets with the Coulombian Model}

This section presents the geometry studied and the model used for the modeling of the torque transmitted between two tile permanent magnets uniformly magnetized. 


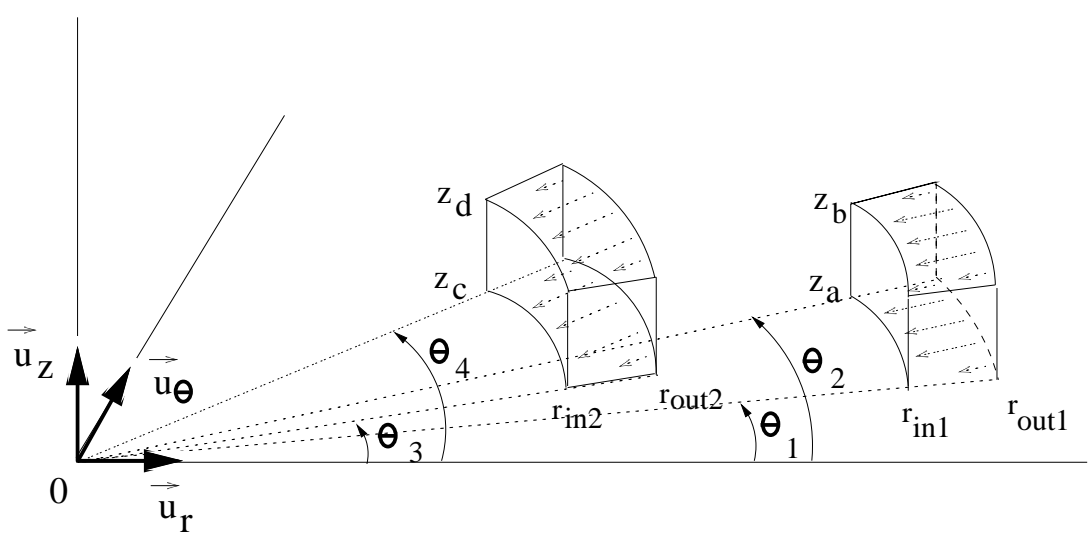

Fig. 1. Representation of the geometry considered in three-dimensions : two tile permanent magnets uniformly magnetized

\section{A. Notation and geometry}

The geometry considered and the related parameters are shown in Fig 1. A two-dimensional repre-

$$
\begin{aligned}
& \overrightarrow{J_{1}}=-\cos \left(\frac{\theta_{2}+\theta_{1}}{2}\right) \vec{u}_{r}-\sin \left(\frac{\theta_{2}+\theta_{1}}{2}\right) \vec{u}_{\theta} \\
& \vec{J}_{2}=-\cos \left(\frac{\theta_{4}+\theta_{3}}{2}\right) \vec{u}_{r}-\sin \left(\frac{\theta_{4}+\theta_{3}}{2}\right) \vec{u}_{\theta}
\end{aligned}
$$

52 The magnetic field created by tile permanent magnets can be obtained by using the Coulombian Model. 53 Indeed, a permanent magnet can be represented by a magnetic pole surface density that is located on 54 the faces of the magnet and a magnetic pole volume density that is located inside the magnet. In the 55 configuration presented in Fig 1, the magnetic pole volume density is 0 because the magnetic polarizations 56 of the magnets are uniform. Consequently, each tile permanent magnet is modelled by its magnetic pole 57 surface density, which is determined as follows: by denoting $\vec{n}_{i j}$, the unit normal vector of the face $i$ of 


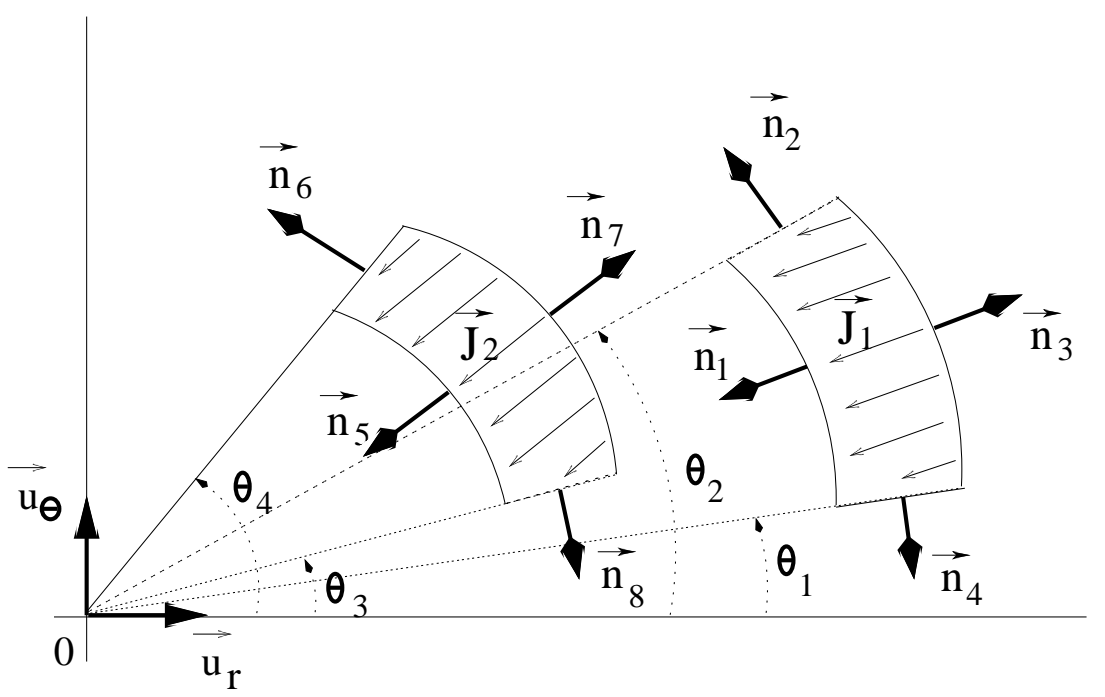

Fig. 2. Representation of the geometry considered in two dimensions : two tile permanent magnets uniformly magnetized

\begin{tabular}{|l|l|c|}
\hline Surface densities & Scalar Product & Expression \\
\hline$\sigma_{11}^{*}$ & $\vec{J}_{1} \cdot \vec{n}_{1}$ & $J_{1} \cos \left(\theta-\frac{\theta_{1}+\theta_{2}}{2}\right)$ \\
\hline$\sigma_{12}^{*}$ & $\vec{J}_{1} \cdot \vec{n}_{2}$ & $J_{1} \sin \left(\frac{\theta_{2}-\theta_{1}}{2}\right)$ \\
\hline$\sigma_{13}^{*}$ & $\vec{J}_{1} \cdot \vec{n}_{3}$ & $-J_{1} \cos \left(\theta-\frac{\theta_{1}+\theta_{2}}{2}\right)$ \\
\hline$\sigma_{14}^{*}$ & $\vec{J}_{1} \cdot \vec{n}_{4}$ & $J_{1} \sin \left(\frac{\theta_{2}-\theta_{1}}{2}\right)$ \\
\hline$\sigma_{25}^{*}$ & $\vec{J}_{2} \cdot \vec{n}_{5}$ & $J_{2} \cos \left(\theta-\frac{\theta_{3}+\theta_{4}}{2}\right)$ \\
\hline$\sigma_{26}^{*}$ & $\vec{J}_{2} \cdot \vec{n}_{6}$ & $J_{2} \sin \left(\frac{\theta_{4}-\theta_{3}}{2}\right)$ \\
\hline$\sigma_{27}^{*}$ & $\vec{J}_{2} \cdot \vec{n}_{7}$ & $-J_{2} \cos \left(\theta-\frac{\theta_{3}+\theta_{4}}{2}\right)$ \\
\hline$\sigma_{28}^{*}$ & $\vec{J}_{2} \cdot \vec{n}_{8}$ & $J_{2} \sin \left(\frac{\theta_{4}-\theta_{3}}{2}\right)$ \\
\hline
\end{tabular}

TABLE I

DEFINITION OF THE MAGNETIC POLE SURFACE DENSITIES LOCATED ON THE MAGNETS

58 the magnet $j$, the corresponding surface density $\sigma_{i j}^{*}$ is determined with (3).

$$
\sigma_{i j}^{*}=\overrightarrow{J_{j}} \cdot \overrightarrow{n_{i j}}
$$

59 Thus, four faces of the two magnets are charged with a magnetic pole surface density. All the surface

60 density calculations are represented in Table I. The torque transmitted between two tile permanent magnets

61 can be determined in two steps. The first step consists in calculating the magnetic field created by one tile

62 permanent magnet. The second step consists in integrating the magnetic field created by the first tile on 
63 the second one. Let us first consider the tile permanent magnet located on the right of Fig 1. By using the

64 Coulombian approach, we can write that the azimuthal field created by this tile is expressed as follows:

$$
\begin{aligned}
H_{\theta}(r, \theta, z)= & \frac{J_{1}}{4 \pi \mu_{0}} \int_{\theta_{1}}^{\theta_{2}} \int_{z_{a}}^{z_{b}} \cos \left(\theta-\frac{\theta_{1}+\theta_{2}}{2}\right) \frac{\vec{u}\left(r_{\text {in } 1}\right)}{\left|\vec{u}\left(r_{\text {in } 1}\right)\right|^{3}} r_{\text {in } 1} d \tilde{\theta} d \tilde{z} \\
& -\frac{J_{1}}{4 \pi \mu_{0}} \int_{\theta_{1}}^{\theta_{2}} \int_{z_{a}}^{z_{b}} \cos \left(\theta-\frac{\theta_{1}+\theta_{2}}{2}\right) \frac{\vec{u}\left(r_{\text {out } 1}\right)}{\left|\vec{u}\left(r_{\text {out } 1}\right)\right|^{3}} r_{\text {out } 1} d \tilde{\theta} d \tilde{z} \\
& +\frac{J_{1}}{4 \pi \mu_{0}} \int_{r_{\text {in } 1}}^{r_{\text {out } 1}} \int_{z_{a}}^{z_{b}} \sin \left(\frac{\theta_{2}-\theta_{1}}{2}\right) \frac{\vec{v}\left(\theta_{2}\right)}{\left|\vec{v}\left(\theta_{2}\right)\right|^{3}} d \tilde{r} d \tilde{z} \\
& +\frac{J_{1}}{4 \pi \mu_{0}} \int_{r_{\text {in } 1}}^{r_{\text {out } 1}} \int_{z_{a}}^{z_{b}} \sin \left(\frac{\theta_{2}-\theta_{1}}{2}\right) \frac{\vec{v}\left(\theta_{1}\right)}{\left|\vec{v}\left(\theta_{1}\right)\right|^{3}} d \tilde{r} d \tilde{z}
\end{aligned}
$$

65 where

$$
\vec{u}(x)=(r-x \cos (\theta-\tilde{\theta})) \vec{u}_{r}-x \sin (\theta-\tilde{\theta}) \vec{u}_{\theta}+(z-\tilde{z}) \vec{u}_{z}
$$

66 and

$$
\vec{v}(y)=\left(r-\tilde{r} \cos (\theta-y) \vec{u}_{r}-\tilde{r} \sin (\theta-y) \vec{u}_{\theta}+(z-\tilde{z}) \vec{u}_{z}\right.
$$

67 The next step is thus to express the torque transmitted to the second tile permanent magnet uniformly 68 magnetized (as shown in Fig 1). By using (4), the torque $T_{\theta}$ can be determined as follows:

$$
\begin{aligned}
T_{\theta}= & \frac{J_{1} J_{2}}{4 \pi \mu_{0}} \int_{\theta_{3}}^{\theta_{4}} \int_{z_{c}}^{z_{d}} r_{\text {in } 2} H\left(r_{\text {in } 2}, \tilde{\tilde{\theta}}, \tilde{\tilde{z}}\right) r_{\text {in } 2} \cos \left(\tilde{\tilde{\theta}}-\frac{\theta_{3}+\theta_{4}}{2}\right) d \tilde{\tilde{\theta}} d \tilde{\tilde{z}} \\
& -\frac{J_{1} J_{2}}{4 \pi \mu_{0}} \int_{\theta_{3}}^{\theta_{4}} \int_{z_{c}}^{z_{d}} r_{\text {out } 2} H\left(r_{\text {out } 2}, \tilde{\tilde{\theta}}, \tilde{\tilde{z}}\right) r_{\text {out } 2} \cos \left(\tilde{\tilde{\theta}}-\frac{\theta_{3}+\theta_{4}}{2}\right) d \tilde{\tilde{\theta}} d \tilde{\tilde{z}} \\
& +\frac{J_{1} J_{2}}{4 \pi \mu_{0}} \int_{r_{\text {in } 2}}^{r_{\text {out } 2}} \int_{z_{c}}^{z_{d}} \tilde{\tilde{r}} H\left(\tilde{\tilde{r}}, \theta_{4}, \tilde{\tilde{z}}\right) \sin \left(\frac{\theta_{4}-\theta_{3}}{2}\right) d \tilde{\tilde{r}} d \tilde{\tilde{z}} \\
& +\frac{J_{1} J_{2}}{4 \pi \mu_{0}} \int_{r_{\text {in } 2}}^{r_{\text {out } 2}} \int_{z_{c}}^{z_{d}} \tilde{\tilde{r}} H\left(\tilde{\tilde{r}}, \theta_{3}, \tilde{\tilde{z}}\right) \sin \left(\frac{\theta_{4}-\theta_{3}}{2}\right) d \tilde{\tilde{r}} d \tilde{\tilde{z}}
\end{aligned}
$$

\section{B. Semi-analytical Expression of the Torque}

The torque transmitted between two tile permanent magnets can be written as follows:

$$
T_{\theta}=\frac{J_{1} J_{2}}{4 \pi \mu_{0}}\left(\int_{\theta_{1}}^{\theta_{2}} \int_{\theta_{3}}^{\theta_{4}} d T_{\theta}^{(1)}+T_{\theta}^{(2)}+\int_{z_{a}}^{z_{b}} d T_{\theta}^{(3)}+\int_{\theta_{1}}^{\theta_{2}} d T_{\theta}^{(4)}\right)
$$




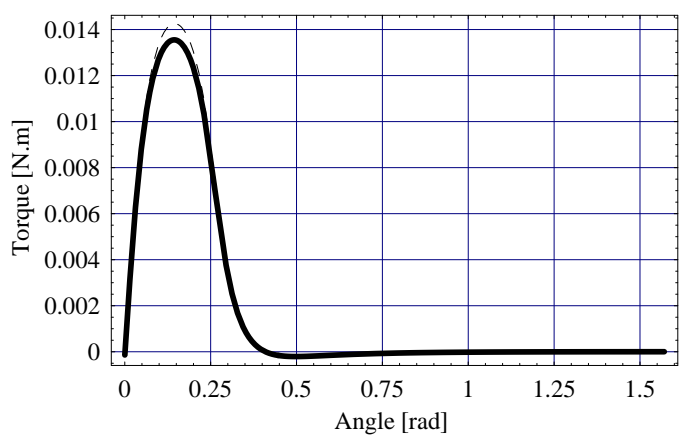

Fig. 3. Representation of the torque transmitted between two tiles ; thick line: the magnetization is uniform, dashed lines: the magnetization is radial; $\theta_{4}-\theta_{3}=\theta_{2}-\theta_{1}=\frac{\pi}{12}, r_{\text {in } 1}=0.025 \mathrm{~m}, r_{\text {out } 1}=0.028 \mathrm{~m}, r_{\text {in } 2}=0.021 \mathrm{~m}, r_{\text {out } 2}=0.024 \mathrm{~m}$, $z_{d}-z_{c}=0.003 \mathrm{~m}, z_{b}-z_{a}=0.003 \mathrm{~m}, z_{a}=0.001 \mathrm{~m}, r=0.024 \mathrm{~m}, J_{1}=J_{2}=1 \mathrm{~T}$

71 where $d T_{\theta}^{(1)}, T_{\theta}^{(2)}, d T_{\theta}^{(3)}$ and $d T_{\theta}^{(4)}$ are given in the appendix. Strictly speaking, this expression is threedimensional and we take into account all the contributions between the two tiles. However, it is noted that all the contributions have not the same weight and the interaction between the surface densities located 74 on the inner and outer faces of each tile permanent magnet is in fact the most important. Furthermore, it 75 is noted that this expression could probably still simplified and led to incomplete elliptical integrals of the ${ }_{76}$ first, second and third kind. In addition, we use the Cauchy principal value for determining the singular 77 cases that appear for example when one tile permanent magnet is exactly in front of the other one.

\section{COMPARISON OF THE TORQUE TRANSMITTED BETWEEN TWO TILES RADIALLY MAGNETIZED AND TWO TILES UNIFORMLY MAGNETIZED}

Tiles uniformly magnetized are generally less efficient than tiles radially magnetized for magnetic couplings. However, this loss of torque depends greatly on the tile angular width. Therefore, it is interesting to determine this loss of torque for different tile angular widths. For this purpose, we represent in the same figure the torque transmitted between two tiles radially magnetized and the torque transmitted between two tiles uniformly magnetized. On Figure 3, the angular width of each tile is the same $\left(\frac{\pi}{12}\right)$. Then, we represent in Figs 4, 5 and 6 the torque transmitted between tiles whose angular widths are $\frac{\pi}{6}, \frac{\pi}{4}$ and $\frac{\pi}{3}$. Figs $3,4,5$ and 6 show clearly that the more the tile angular width is important, the less the magnetic torque between tiles uniformly magnetized is important. Consequently, we deduct that a manufacturer should use tiles uniformly magnetized only if their widths are small. Furthermore, Figs 3, 


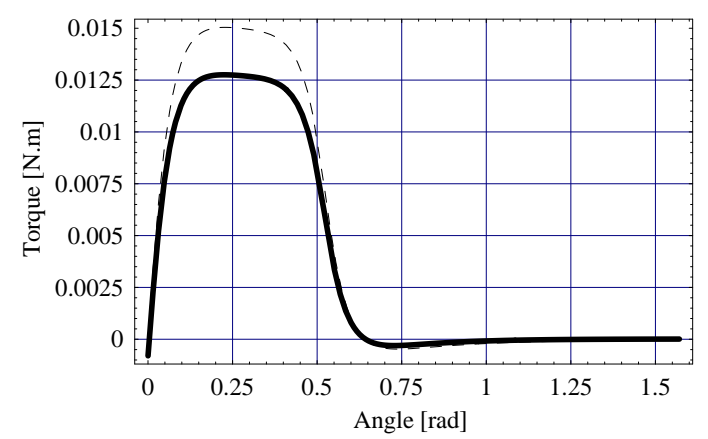

Fig. 4. Representation of the torque transmitted between two tiles ; thick line: the magnetization is uniform, dashed lines: the magnetization is radial; $\theta_{4}-\theta_{3}=\theta_{2}-\theta_{1}=\frac{\pi}{6}, r_{\text {in } 1}=0.025 \mathrm{~m}, r_{\text {out } 1}=0.028 \mathrm{~m}, r_{\text {in } 2}=0.021 \mathrm{~m}, r_{\text {out } 2}=0.024 \mathrm{~m}$, $z_{d}-z_{c}=0.003 \mathrm{~m}, z_{b}-z_{a}=0.003 \mathrm{~m}, z_{a}=0.001 \mathrm{~m}, r=0.024 \mathrm{~m}, J_{1}=J_{2}=1 \mathrm{~T}$

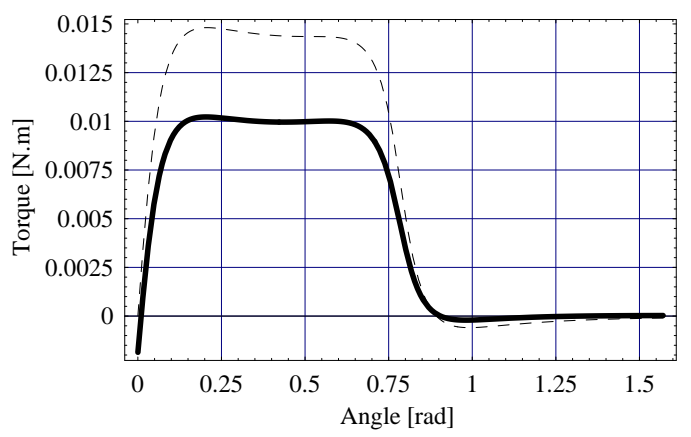

Fig. 5. Representation of the torque transmitted between two tiles ; thick line: the magnetization is uniform, dashed lines: the magnetization is radial; $\theta_{4}-\theta_{3}=\theta_{2}-\theta_{1}=\frac{\pi}{4}, r_{\text {in } 1}=0.025 \mathrm{~m}, r_{\text {out } 1}=0.028 \mathrm{~m}, r_{\text {in } 2}=0.021 \mathrm{~m}, r_{\text {out } 2}=0.024 \mathrm{~m}$, $z_{d}-z_{c}=0.003 \mathrm{~m}, z_{b}-z_{a}=0.003 \mathrm{~m}, z_{a}=0.001 \mathrm{~m}, r=0.024 \mathrm{~m}, J_{1}=J_{2}=1 \mathrm{~T}$

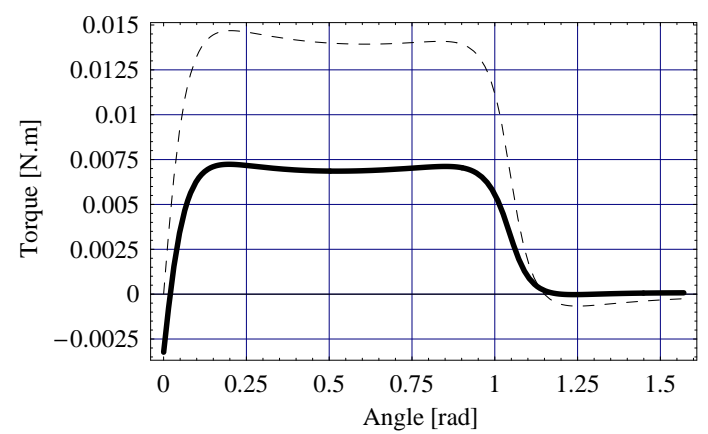

Fig. 6. Representation of the torque transmitted between two tiles ; thick line: the magnetization is uniform, dashed lines: the magnetization is radial; $\theta_{4}-\theta_{3}=\theta_{2}-\theta_{1}=\frac{\pi}{3}, r_{\text {in } 1}=0.025 \mathrm{~m}, r_{\text {out } 1}=0.028 \mathrm{~m}, r_{\text {in } 2}=0.021 \mathrm{~m}, r_{\text {out } 2}=0.024 \mathrm{~m}$, $z_{d}-z_{c}=0.003 \mathrm{~m}, z_{b}-z_{a}=0.003 \mathrm{~m}, z_{a}=0.001 \mathrm{~m}, r=0.024 \mathrm{~m}, J_{1}=J_{2}=1 \mathrm{~T}$ 


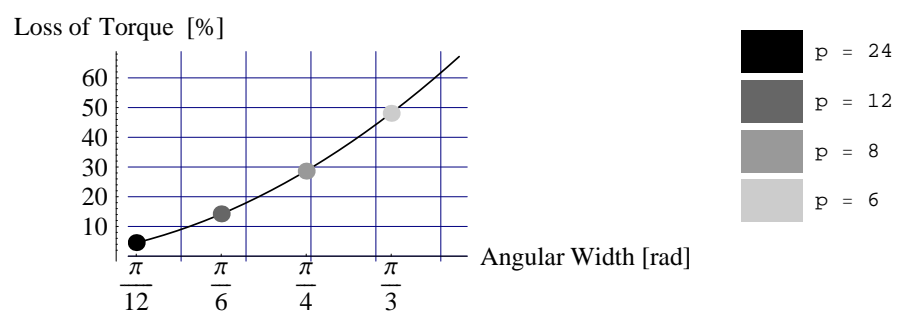

Fig. 7. Representation of the mean loss of efficiency of the torque transmitted between two tiles uniformly magnetized versus the angular width of the tiles. This loss of torque is calculated in comparison with the torque transmitted between two tiles radially magnetized. The points correspond to the figures $3,4,5$ and 6 . We have $: r_{\text {in } 1}=0.025 \mathrm{~m}, r_{\text {out } 1}=0.028 \mathrm{~m}, r_{\text {in } 2}=0.021 \mathrm{~m}$, $r_{\text {out } 2}=0.024 \mathrm{~m}, z_{b}-z_{a}=z_{d}-z_{c}=0.003 \mathrm{~m}, J_{1}=J_{2}=1 \mathrm{~T}$.

4, 5 and 6 show that the torque transmitted between two tile permanent magnets radially magnetized does not depend very much on their angular widths whereas it decreases greatly with the increase in the angular width when the tiles are uniformly magnetized.

We can also represent this decrease by calculating its rate of loss versus the angular width of tiles (Fig 7).

Figure 7 is consistent with Figs 3, 4, 5 and 6. Indeed, when the angular width of the tile permanent magnets tends to zero, we can use either tiles radially magnetized or tiles uniformly magnetized. This is the only case in which the choice has not a great importance. Consequently, as tiles uniformly magnetized are cheaper to fabricate, their use is more judicious. However, when the angular width of the tiles used becomes greater, the choice is certainly more difficult and other considerations must be taken into account. For example, if the first property required in a coupling is really the value of the torque transmitted, the magnetization of the tiles should be radial and not uniform.

\section{APPLICATION : ALTERNATE MAGNET STRUCTURES USING TILES RADIALLY OR UNIFORMLY}

\section{MAGNETIZED}

We can illustrate the expression established in the previous section by studying the torque transmitted in an alternate magnet structure with 8 tile permanent magnets on each rotor. For this purpose, we use the principle of superposition with (7) for the calculation of the torque transmitted between the leading 


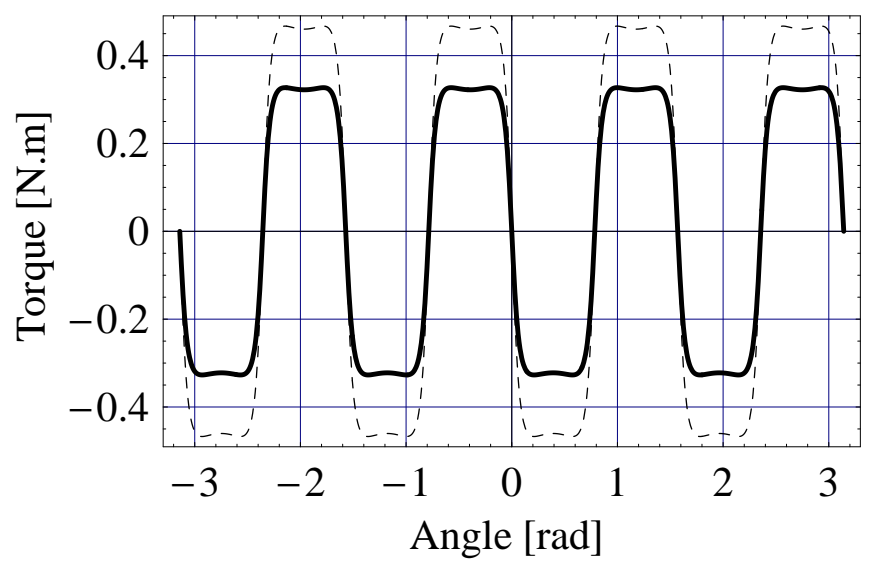

Fig. 8. Representation of the total torque transmitted between an eight tile rotor and an eight tile stator versus the angle $\theta$. The tiles are either radially magnetized (dashed line) or uniformly magnetized (thick line) $r_{\text {in } 1}=0.025 \mathrm{~m}, r_{\text {out } 1}=0.028 \mathrm{~m}$, $r_{\text {in } 2}=0.021 \mathrm{~m}, r_{\text {out } 2}=0.024 \mathrm{~m}, z_{b}-z_{a}=z_{d}-z_{c}=0.003 \mathrm{~m}, J_{1}=J_{2}=1 \mathrm{~T}$

rotor and the lead rotor. We represent this torque versus the shifting angle $\theta$ in Fig 8 .

Figure 8 is consistent with the previous representations of the torque transmitted between two tiles radially or uniformly magnetized. First, we note that the torque transmitted in the alternate magnet structure is sixteen times greater than the one transmitted between only two tile permanent magnets. Then, we note that the torque transmitted between tiles uniformly magnetized is smaller than the one transmitted between tiles radially magnetized, which is still consistent with Fig 4.

\section{CONClusion}

This paper has presented a three-dimensional expression of the torque transmitted between two tile permanent magnets uniformly magnetized. Then, we have compared the torque transmitted between two tiles uniformly magnetized with two tiles radially magnetized. An alternate magnet structure has been studied to illustrate the three-dimensional expression of the torque transmitted between tiles radially or uniformly magnetized. Some theoretical results have been analytically determined. First, tiles uniformly magnetized are less interesting than tiles radially magnetized for realizing couplings. Indeed, the more the tile angular widths are important, the less the torque transmitted between two tiles uniformly magnetized is important in comparison with the torque transmitted between two tiles radially magnetized. However, the cost of the magnets must be taken into account. Tiles uniformly magnetized are easier to fabricate 
than tiles radially magnetized (and thus cheaper...).

We give the expressions of the parameters used for calculating the torque transmitted between two

$$
T_{\theta}=\frac{J_{1} J_{2}}{4 \pi \mu_{0}}\left(\int_{\theta_{1}}^{\theta_{2}} \int_{\theta_{3}}^{\theta_{4}} d T_{\theta}^{(1)}+T_{\theta}^{(2)}+\int_{z_{a}}^{z_{b}} d T_{\theta}^{(3)}+\int_{\theta_{1}}^{\theta_{2}} d T_{\theta}^{(4)}\right)
$$

where

$$
\begin{aligned}
d T_{\theta}^{(1)}= & +\cos \left(\tilde{\theta}-\frac{\theta_{1}+\theta_{2}}{2}\right) \cos \left(\tilde{\tilde{\theta}}-\frac{\theta_{3}+\theta_{4}}{2}\right) \sin (\tilde{\tilde{\theta}}-\tilde{\theta}) A\left[r_{\text {in } 1}, r_{\text {in } 2}\right] d \tilde{\theta} d \tilde{\tilde{\theta}} \\
& -\cos \left(\tilde{\theta}-\frac{\theta_{1}+\theta_{2}}{2}\right) \cos \left(\tilde{\tilde{\theta}}-\frac{\theta_{3}+\theta_{4}}{2}\right) \sin (\tilde{\tilde{\theta}}-\tilde{\theta}) A\left[r_{\text {out } 1}, r_{\text {in } 2}\right] d \tilde{\theta} d \tilde{\tilde{\theta}} \\
& -\cos \left(\tilde{\theta}-\frac{\theta_{1}+\theta_{2}}{2}\right) \cos \left(\tilde{\tilde{\theta}}-\frac{\theta_{3}+\theta_{4}}{2}\right) \sin (\tilde{\tilde{\theta}}-\tilde{\theta}) A\left[r_{\text {in } 1}, r_{\text {out } 2}\right] d \tilde{\theta} d \tilde{\tilde{\theta}} \\
& +\cos \left(\tilde{\theta}-\frac{\theta_{1}+\theta_{2}}{2}\right) \cos \left(\tilde{\tilde{\theta}}-\frac{\theta_{3}+\theta_{4}}{2}\right) \sin (\tilde{\tilde{\theta}}-\tilde{\theta}) A\left[r_{\text {in } 2}, r_{\text {out } 1}\right] d \tilde{\theta} d \tilde{\tilde{\theta}}
\end{aligned}
$$

128

$$
\begin{aligned}
T_{\theta}^{(2)}= & +\sin \left(\frac{\theta_{2}-\theta_{1}}{2}\right) \sin \left(\frac{\theta_{4}-\theta_{3}}{2}\right) B\left[\theta_{2}, \theta_{4}\right] \\
& +\sin \left(\frac{\theta_{2}-\theta_{1}}{2}\right) \sin \left(\frac{\theta_{4}-\theta_{3}}{2}\right) B\left[\theta_{1}, \theta_{4}\right] \\
& +\sin \left(\frac{\theta_{2}-\theta_{1}}{2}\right) \sin \left(\frac{\theta_{4}-\theta_{3}}{2}\right) B\left[\theta_{2}, \theta_{3}\right] \\
& +\sin \left(\frac{\theta_{2}-\theta_{1}}{2}\right) \sin \left(\frac{\theta_{4}-\theta_{3}}{2}\right) B\left[\theta_{1}, \theta_{3}\right]
\end{aligned}
$$

129

$$
\begin{aligned}
d T_{\theta}^{(3)}= & \sin \left(\frac{\theta_{2}-\theta_{1}}{2}\right) \cos \left(\frac{\theta_{2}-\theta_{1}}{2}-\frac{\theta_{4}-\theta_{3}}{2}\right) C\left[\theta_{2}, r_{\text {in } 2}\right] d \tilde{z} \\
& +\sin \left(\frac{\theta_{2}-\theta_{1}}{2}\right) \cos \left(\frac{\theta_{2}-\theta_{1}}{2}-\frac{\theta_{3}+\theta_{4}}{2}\right) C\left[\theta_{1}, r_{\text {in } 2}\right] d \tilde{z} \\
& -\sin \left(\frac{\theta_{2}-\theta_{1}}{2}\right) \cos \left(\frac{\theta_{2}-\theta_{1}}{2}-\frac{\theta_{3}+\theta_{4}}{2}\right) C\left[\theta_{2}, r_{\text {out } 2}\right] d \tilde{z} \\
& -\sin \left(\frac{\theta_{2}-\theta_{1}}{2}\right) \cos \left(\frac{\theta_{2}-\theta_{1}}{2}-\frac{\theta_{3}+\theta_{4}}{2}\right) C\left[\theta_{1}, r_{\text {out } 2}\right] d \tilde{z}
\end{aligned}
$$




$$
\begin{aligned}
d T_{\theta}^{(4)}= & +\cos \left(\tilde{\theta}-\frac{\theta_{1}+\theta_{2}}{2}\right) \sin \left(\frac{\theta_{4}-\theta_{3}}{2}\right) D\left[\theta_{4}, r_{\text {in } 1}\right] d \tilde{\theta} \\
& -\cos \left(\tilde{\theta}-\frac{\theta_{1}+\theta_{2}}{2}\right) \sin \left(\frac{\theta_{4}-\theta_{3}}{2}\right) D\left[\theta_{4}, r_{\text {out } 1}\right] d \tilde{\theta} \\
& +\cos \left(\tilde{\theta}-\frac{\theta_{1}+\theta_{2}}{2}\right) \sin \left(\frac{\theta_{2}-\theta_{1}}{2}\right) D\left[\theta_{3}, r_{\text {in } 1}\right] d \tilde{\theta} \\
& -\cos \left(\tilde{\theta}-\frac{\theta_{1}+\theta_{2}}{2}\right) \sin \left(\frac{\theta_{2}-\theta_{1}}{2}\right) D\left[\theta_{3}, r_{\text {out } 1}\right] d \tilde{\theta}
\end{aligned}
$$

134

35

136

$$
A\left[r_{1}, r_{2}\right]=\int_{z_{a}}^{z_{b}} \int_{z_{c}}^{z_{d}} \frac{-r_{1}^{2} r_{2}^{2}}{\left(r_{1}^{2}+r_{2}^{2}-2 r_{1} r_{2} \cos (\tilde{\tilde{\theta}}-\tilde{\theta})+(\tilde{z}-\tilde{z})^{2}\right)^{\frac{3}{2}}} d \tilde{z} d \tilde{\tilde{z}}
$$

$$
B\left[\theta_{i}, \theta_{j}\right]=\int_{r_{i n 2}}^{r_{\text {out } 2}} \int_{z_{c}}^{z_{d}} \int_{r_{\text {in } 1}}^{r_{\text {out } 1}} \int_{z_{a}}^{z_{b}} \frac{-\tilde{r} \tilde{\tilde{r}} \sin \left(\theta_{j}-\theta_{i}\right)}{\left(\tilde{r}^{2}+\tilde{\tilde{r}}^{2}-2 \tilde{r} \tilde{\tilde{r}} \cos \left(\theta_{j}-\theta_{i}\right)+(\tilde{\tilde{z}}-\tilde{z})^{2}\right)^{\frac{3}{2}}} d \tilde{r} d \tilde{z} d \tilde{\tilde{r}} d \tilde{\tilde{z}}
$$

$$
C\left[\theta_{i}, r_{i}\right]=\int_{r_{\text {in } 1}}^{r_{\text {out } 1}} \int_{z_{a}}^{z_{b}} \int_{z_{c}}^{z_{d}} \frac{-r_{i}^{2} \tilde{r} \sin \left(\tilde{\tilde{\theta}}-\theta_{i}\right)}{\left(\tilde{r}^{2}+r_{i}^{2}-2 \tilde{r} r_{i} \cos \left(\tilde{\tilde{\theta}}-\theta_{i}\right)+(\tilde{\tilde{z}}-\tilde{z})^{2}\right)^{\frac{3}{2}}} d \tilde{r} d \tilde{z} d \tilde{\tilde{z}}
$$

$$
D\left[\theta_{j}, r_{j}\right]=\int_{r_{\text {in } 2}}^{r_{\text {out } 2}} \int_{z_{c}}^{z_{d}} \int_{z_{a}}^{z_{b}} \frac{-r_{j}^{2} \tilde{\tilde{r}} \sin \left(\theta_{j}-\theta_{i}\right)}{\left(\tilde{\tilde{r}}^{2}+r_{j}^{2}-2 r_{j} \tilde{r} \cos \left(\theta_{j}-\tilde{\theta}\right)+(\tilde{\tilde{z}}-\tilde{z})^{2}\right)^{\frac{3}{2}}} d \tilde{z} d \tilde{\tilde{r}} d \tilde{\tilde{z}}
$$

The torque transmitted between two tile permanent magnets could be calculated directly by numerical means with (8). However, the computational cost would be too long. Therefore, we give here four reduced semi-analytical expressions of $A\left[r_{1}, r_{2}\right], B\left[\theta_{i}, \theta_{j}\right], C\left[\theta_{i}, r_{i}\right]$ and $D\left[\theta_{j}, r_{j}\right]$. We obtain:

$$
A\left[r_{1}, r_{2}\right]=A^{(1)}\left[z_{a}, z_{c}\right]-A^{(1)}\left[z_{b}, z_{c}\right]-A^{(1)}\left[z_{a}, z_{d}\right]+A^{(1)}\left[z_{b}, z_{d}\right]
$$

with

$$
A^{(1)}\left[z_{i}, z_{j}\right]=\frac{r_{1}^{2} r_{2}^{2} \sqrt{r_{1}^{2}+r_{2}^{2}+\left(z_{i}-z_{j}\right)^{2}-2 r_{1} r_{2} \cos (\tilde{\theta}-\tilde{\tilde{\theta}})}}{r_{1}^{2}+r_{2}^{2}-2 r_{1} r_{2} \cos (\tilde{\theta}-\tilde{\tilde{\theta}})}
$$


141

$$
\begin{aligned}
B\left[\theta_{i}, \theta_{j}\right]= & \int_{r_{\text {in } 2}}^{r_{\text {out } 2}}\left(-B^{(1)}\left[z_{a}-z_{c}, r_{\text {out } 1}\right]+B^{(1)}\left[z_{b}-z_{c}, r_{\text {out } 1}\right]\right) d \tilde{\tilde{r}} \\
& +\int_{r_{\text {in } 2}}^{r_{\text {out } 2}}\left(+B^{(1)}\left[z_{a}-z_{d}, r_{\text {out } 1}\right]-B^{(1)}\left[z_{b}-z_{d}, r_{\text {out } 1}\right]\right) d \tilde{\tilde{r}} \\
& +\int_{r_{\text {in } 2}}^{r_{\text {out } 2}}\left(B^{(1)}\left[z_{a}-z_{c}, r_{\text {in } 1}\right]-B^{(1)}\left[z_{b}-z_{c}, r_{\text {in } 1}\right]\right) d \tilde{\tilde{r}} \\
& +\int_{r_{\text {in } 2}}^{r_{\text {out } 2}}\left(-B^{(1)}\left[z_{a}-z_{d}, r_{\text {in } 1}\right]+B^{(1)}\left[z_{b}-z_{d}, r_{\text {in } 1}\right]\right) d \tilde{\tilde{r}}
\end{aligned}
$$

142

with

$$
\begin{aligned}
B\left[y, r_{i}\right]= & \tilde{\tilde{r}} \sqrt{\tilde{r}^{2}-2 \tilde{r} \tilde{\tilde{r}} x+y}+\tilde{\tilde{r}}^{2} x \log \left[\tilde{r}-\tilde{\tilde{r}} x+\sqrt{\tilde{r}^{2}-2 \tilde{r} \tilde{\tilde{r}} x+y}\right] \\
& +\frac{\tilde{\tilde{r}} i\left(-x+\sqrt{-1+x^{2}} \sqrt{\tilde{\tilde{r}}^{2}-y}\right)}{2 \sqrt{-1+x^{2}}} \log [A] \\
& +\frac{\tilde{\tilde{r}} i\left(x+\sqrt{-1+x^{2}}\right) \sqrt{\tilde{\tilde{r}}^{2}-y}}{2 \sqrt{-1+x^{2}}} \log [B]
\end{aligned}
$$

143

$$
\begin{aligned}
A= & \frac{2 i\left(\tilde{r} \tilde{\tilde{r}}\left(-1+x^{2}\right)+\tilde{\tilde{r}}^{2}\left(x-x^{3}+x^{2} \sqrt{-1+x^{2}}\right)\right)}{\left(-x+\sqrt{-1+x^{2}}\right)\left(\tilde{r}+\tilde{\tilde{r}}\left(-x+\sqrt{-1+x^{2}}\right)\right)\left(\tilde{\tilde{r}}^{2}-y\right)^{\frac{3}{2}}} \\
& +\frac{2 i \sqrt{-1+x^{2}}\left(-y+i \sqrt{\tilde{\tilde{r}}^{2}-y} \sqrt{\tilde{r}^{2}-2 \tilde{r} \tilde{r} x+y}\right)}{\left(-x+\sqrt{-1+x^{2}}\right)\left(\tilde{r}+\tilde{\tilde{r}}\left(-x+\sqrt{-1+x^{2}}\right)\right)\left(\tilde{\tilde{r}}^{2}-y\right)^{\frac{3}{2}}}
\end{aligned}
$$

144

$$
\begin{aligned}
B= & \frac{2 i \tilde{r} \tilde{\tilde{r}}\left(-1+x^{2}\right)-2 i \tilde{\tilde{r}}^{2} x\left(-1+x^{2}+x \sqrt{-1+x^{2}}\right)}{\left(x+\sqrt{-1+x^{2}}\right)(-\tilde{r}+\tilde{\tilde{r}})\left(x+\sqrt{-1+x^{2}}\right)\left(\tilde{\tilde{r}}^{2}-y\right)^{\frac{3}{2}}} \\
& +\frac{2 \sqrt{-1+x^{2}}\left(i y+\sqrt{\tilde{\tilde{r}}^{2}-y} \sqrt{\tilde{r}^{2}-2 \tilde{r} \tilde{r} x+y}\right)}{\left(x+\sqrt{-1+x^{2}}\right)\left(-\tilde{r}+\tilde{\tilde{r}}\left(x+\sqrt{-1+x^{2}}\right)\right)\left(\tilde{\tilde{r}}^{2}-y\right)^{\frac{3}{2}}}
\end{aligned}
$$

145

$$
x=\cos \left(\theta_{i}-\theta_{j}\right)
$$




$$
\begin{aligned}
& C\left[\theta_{i}, r_{i}\right]=\int_{r_{\text {in } 1}}^{r_{\text {out } 1}} C^{(1)}\left[\theta_{i}, r_{i}\right] d \tilde{r} \\
& C^{(1)}\left[\theta_{i}, r_{i}\right]=r_{i} \sqrt{r_{i}^{2}+\tilde{r}^{2}+\left(z_{c}-\tilde{z}\right)^{2}-2 r_{i} \tilde{r} \cos \left(\theta_{3}-\theta_{i}\right)} \\
& -r_{i} \sqrt{r_{i}^{2}+\tilde{r}^{2}+\left(z_{d}-\tilde{z}\right)^{2}-2 r_{i} \tilde{r} \cos \left(\theta_{3}-\theta_{i}\right)} \\
& -r_{i} \sqrt{r_{i}^{2}+\tilde{r}^{2}+\left(z_{c}-\tilde{z}\right)^{2}-2 r_{i} \tilde{r} \cos \left(\theta_{4}-\theta_{i}\right)} \\
& +r_{i} \sqrt{r_{i}^{2}+\tilde{r}^{2}+\left(z_{d}-\tilde{z}\right)^{2}-2 r_{i} \tilde{r} \cos \left(\theta_{4}-\theta_{i}\right)} \\
& +r_{i}\left(z_{c}-\tilde{z}\right) \log \left[-z_{c}+\tilde{z}+\sqrt{r_{i}^{2}+\tilde{r}^{2}+\left(z_{c}-\tilde{z}\right)^{2}-2 r_{i} \tilde{r} \cos \left(\theta_{3}-\theta_{i}\right)}\right] \\
& -r_{i}\left(z_{d}-\tilde{z}\right) \log \left[-z_{d}+\tilde{z}+\sqrt{r_{i}^{2}+\tilde{r}^{2}+\left(z_{d}-\tilde{z}\right)^{2}-2 r_{i} \tilde{r} \cos \left(\theta_{4}-\theta_{i}\right)}\right] \\
& +r_{i}\left(z_{d}-\tilde{z}\right) \log \left[-z_{d}+\tilde{z}+\sqrt{r_{i}^{2}+\tilde{r}^{2}+\left(z_{d}-\tilde{z}\right)^{2}-2 r_{i} \tilde{r} \cos \left(\theta_{3}-\theta_{i}\right)}\right] \\
& -r_{i}\left(z_{c}-\tilde{z}\right) \log \left[-z_{c}+\tilde{z}+\sqrt{r_{i}^{2}+\tilde{r}^{2}+\left(z_{c}-\tilde{z}\right)^{2}-2 r_{i} \tilde{r} \cos \left(\theta_{3}-\theta_{i}\right)}\right] \\
& D\left[\theta_{j}, r_{j}\right]=\int_{r_{\text {in } 2}}^{r_{\text {out } 2}}\left(D^{(2)}\left(z_{b}, z_{c}\right)-D^{(2)}\left(z_{a}, z_{c}\right)+D^{(2)}\left(z_{a}, z_{d}\right)-D^{(2)}\left(z_{b}, z_{d}\right)\right) d \tilde{\tilde{r}}
\end{aligned}
$$

146 with

$$
D^{(2)}(x, y)=\frac{\sin \left(\theta_{i}-\theta_{j}\right) r_{j}^{2} \tilde{\tilde{r}} \sqrt{r_{j}^{2}+\tilde{\tilde{r}}^{2}+(x-y)^{2}-2 r_{j} \tilde{\tilde{r}} \cos \left(\theta_{j}-\theta_{i}\right)}}{r_{j}^{2}+\tilde{\tilde{r}}^{2}-2 r_{j} \tilde{\tilde{r}} \cos \left(\theta_{j}-\theta_{i}\right)}
$$

\section{REFERENCES}

[1] J. P. Yonnet, "Permanent magnet bearings and couplings," IEEE Trans. Magn., vol. 17, pp. 1169-1173, Jan 1981.

[2] J. P. Yonnet, Rare-earth Iron Permanent Magnets, ch. Magnetomechanical devices. Oxford science publications, 1996.

[3] J.P.Yonnet, S. Hemmerlin, E. Rulliere, and G. Lemarquand, "Analytical calculation of permanent magnet couplings," IEEE Trans. Magn., vol. 29, pp. 2932-2934, Nov. 1993.

[4] P. Elies and G. Lemarquand, "Analytical study of radial stability of permanent magnet synchronous couplings," IEEE Trans. Magn., vol. 35, pp. 2133-2136, July 1999.

[5] J. P. Yonnet, "Passive magnetic bearings with permanent magnets," IEEE Trans. Magn., vol. 14, pp. 803-805, Sept. 1978. 
[6] J. Delamare, E. Rulliere, and J. Yonnet, "Classification and synthesis of permanent magnet bearing configurations," IEEE. Trans. Magn., vol. 31, pp. 4190-4192, Nov. 1995.

[7] G. Lemarquand, "Ironless loudspeakers," IEEE Trans. Magn., vol. 43, pp. 3371-3374, August 2007.

[8] P. Samanta and H. Hirani, "Magnetic bearing configurations: Theoretical and experimental studies," IEEE Trans. Magn., vol. 44, pp. 292-300, Feb. 2008.

[9] S. Mukhopadhyay, J. Donaldson, G. Sengupta, S. Yamada, C. Chakraborty, and D. Kacprzak, "Fabrication of a repulsive-type magnetic bearing using a novel arrangement of permanent magnets for vertical-rotor suspension," IEEE Trans. Magn., vol. 39, pp. 3220-3222, Sept. 2003.

[10] R. Moser, J. Sandtner, and H. Bleuler, "Optimization of repulsive passive magnetic bearings," IEEE. Trans. Magn., vol. 42, pp. 2038-2042, Aug. 2006.

[11] F. Bancel and G. Lemarquand, "Three-dimensional analytical optimization of permanent magnets alternated structure," IEEE Trans. Magn., vol. 34, pp. 242-247, Jan. 1998.

[12] V. Lemarquand, J. F. Charpentier, and G. Lemarquand, "Nonsinusoidal torque of permanent-magnet couplings," IEEE Trans. Magn., vol. 35, no. 5, pp. 4200-4205, 1999.

[13] J. Charpentier and G. Lemarquand, "Optimization of unconventional p.m. couplings," IEEE Trans. Magn., vol. 38, pp. 10931096, March 2002.

[14] G. Akoun and J. P. Yonnet, "3d analytical calculation of the forces exerted between two cuboidal magnets," IEEE Trans. Magn., vol. 20, no. 5, pp. 1962-1964, 1984.

[15] J. Hilton and S. McMurry, "Halbach cylinders with improved field homogeneity and tailored gradient fields," IEEE Trans. Magn., vol. 43, pp. 1898-1902, may 2007.

[16] K. Halbach, "Design of permanent multiple magnets with oriented rec material," Nucl. Inst. Meth., vol. 169, pp. 1-10, 1980.

[17] M. Abele and H. A. Leupold, "A general method for flux confinement in permanent magnet structure," J. Appl. Phys., vol. 64, no. 10 , pp. 5988-5990, 1988.

[18] O. M. Kwon, C. Surussavadee, M. Chari, S. Salon, and K. S.vasubramaniam, "Analysis of the far field of permanent magnet motors and effects of geometric asymmetries and unbalance in magnet design," IEEE Trans. Magn., vol. 40, pp. 435-442, march 2004.

[19] M. Abele, J. Jensen, and H. Rusinek, "Generation of uniform high fields with magnetized wedges," IEEE Trans. Magn., vol. 33, no. 5, pp. 3874-3876, 1997.

[20] R. Ravaud, G. Lemarquand, V. Lemarquand, and C. Depollier, "Analytical calculation of the magnetic field created by permanent-magnet rings," IEEE Trans. Magn., vol. 44, pp. 1982-1989, Aug 2008.

[21] J. Conway, "Inductance calculations for noncoaxial coils using bessel functions," IEEE Trans. Magn., vol. 43, pp. 1023-1034, march 2007.

[22] J. Conway, "Noncoaxial inductance calculations without the vector potential for axisymmetric coils and planar coils," IEEE Trans. Magn., vol. 44, pp. 453-462, oct. 2008.

[23] K. Kim, E. Levi, Z. Zabar, and L. Birenbaum, "Mutual inductance of noncoaxial circular coils with constant current density," IEEE Trans. Magn., vol. 33, pp. 4303-4309, sept. 1997. 
[24] S. Babic and C. Akyel, "Magnetic force calculation between thin coaxial circular coils in air," IEEE Trans. Magn., vol. 44, pp. 445-452, April 2008.

[25] S. Babic, C. Akyel, S. Salon, and S. Kincic, "New expressions for calculating the magnetic field created by radial current in massive disks," IEEE Trans. Magn., vol. 38, pp. 497-500, march 2002.

[26] S. Babic and M. Gavrilovic, "New expression for calculating magnetic fields due to current-carrying solid conductors," IEEE Trans. Magn., vol. 33, pp. 4134-4136, may 1997.

[27] S. Babic and C. Akyel, "An improvement in the calculation of the magnetic field for an arbitrary geometry coil with rectangular cross section," International Journal of Numerical Modelling: Electronic Networks, Devices and Fields, vol. 18, pp. 493-504, November 2005.

[28] S. Babic and C. Akyel, "Calculating mutual inductance between circular coils with inclined axes in air," IEEE Trans. Magn., vol. 44, pp. 1743-1750, July 2008.

[29] J. P. Selvaggi, S. Salon, O. -M. Kwon, and M. V. K. Chari, "Calculating the external magnetic field from permanent magnets in permanent-magnet motors - an alternative method," IEEE Trans. Magn., vol. 40, pp. 3278-3285, Sept. 2004.

[30] J. P. Selvaggi, S. Salon, O. -M. Kwon, M. V. K. Chari, and M. DeBortoli, "Computation of the external magnetic field, near-field or far-field from a circular cylindrical magnetic source using toroidal functions," IEEE Trans. Magn., vol. 43, pp. 1153-1156, Avril 2007.

[31] E. P. Furlani, "Formulas for the force and torque of axial couplings," IEEE Trans. Magn., vol. 29, pp. 2295-2301, Sept. 1993.

[32] E. Furlani, S. Reznik, and A. Kroll, "A three-dimensonal field solution for radially polarized cylinders," IEEE Trans. Magn., vol. 31, pp. 844-851, Jan. 1995.

[33] E. Furlani, "Field analysis and optimization of ndfeb axial field permanent magnet motors," IEEE Trans. Magn., vol. 33, pp. 3883-3885, Sept. 1997.

[34] B. Azzerboni, E. Cardelli, and A. Tellini, "Computation of the magnetic field in massive conductor systems," IEEE Trans. Magn., vol. 25, pp. 4462-4473, november 1989.

[35] B. Azzerboni and E. Cardelli, "Magnetic field evaluation for disk conductors," IEEE Trans. Magn., vol. 29, pp. 2419-2421, november 1993.

[36] B. Azzerboni and G. Saraceno, "Three-dimensional calculation of the magnetic field created by current-carrying massive disks," IEEE Trans. Magn., vol. 34, pp. 2601-2604, september 1998.

[37] E. Perigo, R. Faria, and C. Motta, "General expressions for the magnetic flux density produced by axially magnetized toroidal permanent magnets," IEEE Trans. Magn., vol. 43, pp. 3826-3832, oct. 2008.

[38] H. Rakotoarison, J. Yonnet, and B. Delinchant, "Using coulombian approach for modeling scalar potential and magnetic field of a permanent magnet with radial polarization," IEEE Trans. Magn., vol. 43, no. 4, pp. 1261-1264, 2007.

[39] Y. Zhilichev, "Calculation of magnetic field of tubular permanent magnet assemblies in cylindrical bipolar coordinates," IEEE Trans. Magn., vol. 43, pp. 3189-3195, July 2007.

[40] E. Durand, "Electrostatique," Masson Editeur, Paris, France, vol. 1, pp. 248-251, 1964.

[41] T. Ohji, C. Mukhopadhyay, M. Iwahara, and S. Yamada, "Performance of repulsive type magnetic bearing system under nonuniform magnetization of permanent magnet," IEEE Trans. Magn., vol. 36, pp. 3696-3698, Sept. 2000. 
[42] T. Ohji, S. Ichiyama, K. Amei, M. Sakui, and S. Yamada, "Conveyance test by oscillation and rotation to a permanent magnet repulsive-type conveyoer," IEEE Trans. Magn., vol. 40, pp. 3057-3059, April 2004.

[43] M. Berkouk, V. Lemarquand, and G. Lemarquand, "Analytical calculation of ironless loudspeaker motors," IEEE Trans. Magn., vol. 37, pp. 1011-1014, March 2001.

[44] L. Yong, Z. Jibin, and L. Yongping, "Optimum design of magnet shape in permanent-magnet synchronous motors," IEEE Trans. Magn., vol. 39, pp. 3523-4205, nov. 2003.

[45] O. Cuguat, J. Delamare, and G. Reyne, "Magnetic micro-actuators and systems (magmas)," IEEE Trans. Magn., vol. 39, pp. 3607-3612, nov 2003.

[46] J. Wang, G. W. Jewell, and D. Howe, "Design optimisation and comparison of permanent magnet machines topologies," vol. 148, pp. 456-464, IEE.Proc.Elect.Power Appl., Sept 2001.

[47] A. A. Hussien, S. Yamada, M. Iwahara, T. Okada, and T. Ohji, "Application of the repulsive-type magnetic bearing for manufacturing micromass measurement balance equipment," IEEE Trans. Magn., vol. 41, pp. 3802-3804, Oct. 2005.

[48] M. Aydin, Z. Zhu, T. Lipo, and D. Howe, "Minimization of cogging torque in axial-flux permanent-magnet machines: design concepts," IEEE Trans. Magn., vol. 43, pp. 3614-3622, sept. 2007. 